\title{
Exposição ao chumbo: consequências neuropsiquiátricas e comportamentais
}

\author{
Exposure to lead: Neuropsychiatric and Behavioral Consequences
}

Mário Francisco P. Juruena

\begin{abstract}
RESUMO
Em praticamente todo tipo de exposição a chumbo, considerando a duração a curto ou longo prazos, em altas ou baixas concentrações, o órgão crítico alvo é sempre o cérebro, promovendo sinais e sintomas tais como, cefaléia, perda de memória, perda da concentração e atenção em tarefas corriqueiras, alterações de humor, com irritabilidade, depressão, insônia (ou sonolência excessiva). Alguns estudos têm demonstrado evidências de que, mesmo em níveis de exposição relativamente baixos, o Chumbo $(\mathrm{Pb})$ detectável foi associado com déficit nas funções cognitivas, intelectuais e funcionamento executivo, comportamentos antissociais, e transtorno de déficit de atenção e hiperatividade déficit. Outros estudos foram capazes de demonstrar que a redução dos níveis de exposição ao $\mathrm{Pb}$ pode ser responsável pela redução dos níveis de impulsividade, agressividade e criminalidade em adolescentes. Por fim evidências suportam a hipótese de que as exposições ao $\mathrm{Pb}$ podem perturbar o desenvolvimento do SNC, e estão associadas com esquizofrenia e transtornos de psicoses relacionados. Alguns estudos que relatam uma relação estrutura-função e psicopatologia são abordados nesta revisão.
\end{abstract}

Palavras-chave: Chumbo/Grau de Concentração. Desempenho Cognitivo. Transtornos Mentais. Psicopatologia/Comportamento.

\section{Introdução}

O chumbo $(\mathrm{Pb})$ é uma das mais antigas e estabelecidas substâncias toxicas. ${ }^{1}$ Os conhecimentos gerais dos seus efeitos tóxicos são descritos há mais de três mil anos e o conhecimento de seus efeitos nas crianças há mais de 100 anos. Sendo por exposição continuada um crescente problema de saúde pública, especialmente nos grandes centros urbanos no EUA e também em nações do Terceiro Mundo. ${ }^{1}$ Ao longo dos últimos 25 anos, tem havido grande interesse e investigação sobre as ligações entre a exposição ao $\mathrm{Pb}$ e os quadros subclínicos e o ajuste cognitivo e comportamental das crianças e dos jovens.

Needleman e colegas ${ }^{2-4}$ foram capazes de demonstrar que, mesmo em níveis de exposição relativamente baixos, o $\mathrm{Pb}$ detectável foi associado com déficit de inteligência e aumento dos níveis de com-
MD, MSc, MPhil, PhD. Departamento de Neurociências e Ciências do Comportamento. Faculdade de Medicina de Ribeirão Preto Universidade de São Paulo
Correspondência: Universidade de São Paulo- Saude Mental Av. Tenente Catão Roxo 2650 14051-140 - Ribeirão Preto Phone/Fax: +55(16)36307961 e-mail: juruena@fmrp.usp.br

Artigo recebido em 05/07/2009 Aprovado em 08/12/2009 
portamento hiperativo. Apesar da controvérsia em torno dos trabalhos de Needleman e col, ${ }^{5-7}$ estas conclusões foram confirmadas por um número crescente de estudos, levando a um consenso de que a exposição a baixos níveis de $\mathrm{Pb}$ tem realmente potencial neurotóxico. ${ }^{8-11}$ Outros estudos sugerem que os déficits observados na infância são observados também na adolescência e no início da idade adulta. ${ }^{12-13}$

Investigações sobre a exposição ao $\mathrm{Pb}$ têm demonstrado que existe associação com déficit de inteligência e de níveis mais elevados de comportamento hiperativo. ${ }^{14}$ Mais recentemente, tem havido sugestão de que a redução dos níveis de exposição ao $\mathrm{Pb}$ pode ser responsável pela redução dos níveis de criminalidade. ${ }^{15}$ Recentemente, os efeitos da exposição ao chumbo e as ligações entre fatores que levam ao aumento nos níveis de crime foi descrito. ${ }^{15}$ Nevin e cols ${ }^{14-15}$ examinaram as relações entre exposição ao $\mathrm{Pb}$ criminalidade violenta na EUA e em uma série de outros países ocidentais, durante a segunda metade do século 20. Foram encontradas associações entre a redução na média dos níveis sanguíneos de $\mathrm{Pb}$ e nas taxas de criminalidade violenta em EUA, concluiu-se então que, as reduções nos níveis de exposição ao $\mathrm{Pb}$ poderiam levar a redução no nível de criminalidade violenta em cada um dos países estudados. ${ }^{15}$ Especificamente, os estudos entre as ligações entre a exposição ao $\mathrm{Pb}$ e o crime podem resultar de um processo cíclico onde à exposição pode levar ao insucesso educacional, com o insucesso educacional, por sua vez, aumentando o risco de cometer crimes. Estas conclusões foram consenso indicando, em uma série de estudos, a relação entre a exposição ao chumbo com comportamentos agressivos e delinquência. ${ }^{16,17,18} \mathrm{~A}$ exposição ao $\mathrm{Pb}$ também inclui déficit nas funções intelectuais e funcionamento executivo, comportamentos antissociais, e transtorno de déficit de atenção e hiperatividade (TDAH). Alguns estudos relatam uma relação estrutura-função, o que traduz, em última instância, como o sistema nervoso central (SNC) responde a função alterada pela exposição ao $\mathrm{Pb}$, podendo perturbar o desenvolvimento do SNC, e associarem-se com esquizofrenia e transtornos de psicoses, conforme vamos abordar nesta revisão.

\section{Funções Cognitivas}

Apesar da bem estabelecida ligação entre exposição ao $\mathrm{Pb}$ levar a déficits cognitivos, poucos estudos têm analisado a associação direta entre a exposi- ção na infância e posteriores lesões na estrutura do SNC com déficit nas funções cognitivas na vida adulta.

Um estudo mostrou que a maior média de concentração sanguínea de chumbo na infância está associada a reduções específicas no volume da substância cinzenta. Os achados sugerem que a exposição ao $\mathrm{Pb}$ na infância está associada com perda de volume considerável em porções do córtex prefrontal e sistema límbico, com alterações nos processos cognitivos e emocionais. ${ }^{19}$

Outras funções cognitivas que se apresentaram alteradas incluem: a antecipação das tarefas cognitivamente exigentes, acompanhamento e conclusão de novos comportamentos, impedindo detecção de erros, avaliação de potenciais conflitos, controle motor complexo, motivação e recompensa com base em decisão tomada. ${ }^{20,21,22}$

Evidência de estudos comportamentais e coortes epidemiológicas, e estudos em modelos animais indicam que indivíduos do sexo masculino são mais vulneráveis aos efeitos do chumbo na função executiva. $^{23,24}$

No entanto, há variação na medida em que a exposição $\mathrm{Pb}$ nestes diversos estudos se confunde com outros fatores de risco para o desenvolvimento cognitivo prejudicado (por exemplo, o baixo QI materno, ambiente doméstico conturbado), bem como os métodos utilizados para avaliar e controlar estatisticamente para estes fatores. Por exemplo, em estudos onde indicadores sensíveis desses fatores não foram incluídos nas análises, as correlações observadas entre exposição $\mathrm{Pb}$ e problemas comportamentais são possivelmente falsas positivas..$^{21,22,23}$

Além da avaliação dos efeitos sobre a cognição, tem havido um interesse crescente nas influências do envenenamento pelo $\mathrm{Pb}$ nos subsequentes estágios de desenvolvimento social/emocional. Sciarillo e colegas ${ }^{25}$ observaram que, em meninos e meninas de 4-5 anos de idade, houve um aumento da incidência de uma variedade de psicopatologias (por exemplo, depressão, somatizações); as crianças expostas apresentaram agressividade e os aumentos de $\mathrm{Pb}$ no sangue foram observados em nível superior a 15 microgramas/ $\mathrm{dl}^{25}$. Needleman e colegas ${ }^{26}$ relataram que, em meninos de 7 anos a associação entre o Pb e comportamento antissocial/delinquente foi limítrofe, mas significativa em amostra de meninos com 11 anos. O primeiro estudo prospectivo longitudinal de $\mathrm{Pb}$ e exposição no período pré-natal e delinquência juvenil mostrou um aumento de comportamentos antissociais 
mesmo com baixo nível de exposição ao $\mathrm{Pb}$. Neste sentido, o ajuste de covariáveis foi fundamental como variáveis médicas (por exemplo, peso ao nascer, Apgar, uso de tranquilizantes durante a gravidez) e sociais (por exemplo QI materno e/ou nível de instrução do cuidados primário). ${ }^{27}$ Embora a exposição ao $\mathrm{Pb}$ esteja associada a aumento de comportamento psicopatólogico, não é claro se os comportamentos observados são causados diretamente pelo $\mathrm{Pb}$ ou são secundários às desvantagens impostas pelas disfunções cognitivas.

\section{Impulsividade, Déficit de Atenção}

Déficit de Atenção é comumente citado como uma consequência de longo prazo da exposição precoce ao $\mathrm{Pb} .{ }^{28-30} \mathrm{De}$ fato, tem sido sugerido que a exposição ao $\mathrm{Pb}$ pode ser assintomática e provocar um TDAH, uma síndrome caracterizada pela deficiente resposta ao meio e atenção comprometida. ${ }^{28} \mathrm{~A}$ maior incidência de TDAH e comportamentos antissociais em pacientes do sexo masculino associa-se de modo consistente com uma maior suscetibilidade e exposição ao Pb na infância. ${ }^{30}$ Froehlich e colegas. ${ }^{31}$ descobriram que a função executiva decorre de uma interação com os receptores dopaminérgicos D4 gene (DRD4-7), sendo o efeito maior no sexo masculino. Braun e seus colegas descobriram que crianças com níveis sanguíneo superior a $2 \mathrm{l}$ g/dl $(0,097 \mathrm{lmol} / \mathrm{l})$, representando o quintil superior de 4-15 anos nos Estados Unidos, apresentaram quatro vezes mais probabilidades de ter diagnóstico de TDAH. ${ }^{32}$ Mais estudos são necessários para reproduzir estes resultados e prestar esclarecimentos sobre a interação gene-ambiente associados com maior vulnerabilidade do sexo masculino a exposição ao $\mathrm{Pb}$.

\section{Violência e criminalidade}

$\mathrm{Na}$ tentativa de identificar um comportamento de exposição ao $\mathrm{Pb}$, Lanphear et al. ${ }^{36}$ sugeriu quatro domínios, atenção, função executiva, integração visual-motora, e comportamento social, todos os quais foram relacionados com exposição ao $\mathrm{Pb}$. Dietrich et al. ${ }^{27}$ sugere um comportamento semelhante na exposição de chumbo, acrescentando domínios da coordenação motora fina e equilíbrio.

Alguns estudos têm relatado comportamento antissocial, problemas nos domínios da delinquência juvenil, comportamento, e de agressão, estes estu- dos avaliaram as crianças muito mais de 7,5 anos de idade 27,34 .

Mendelsohn et al. ${ }^{34}$ concluiu que crianças expostas ao $\mathrm{Pb}$ tinham mais dificuldades na regulação das emoções e comportamento sexual. Estas crianças foram mais hiperativas, impulsivas e facilmente frustradas, e tinham mais comportamentos de desinteresse. Assim, a exposição ao $\mathrm{Pb}$ parece estar associada ao aumento de psicopatologias na infância e que, na adolescência, muitas crianças expostas podem se tornar agressivos, antissociais, e delinquentes.

Existe também indicativo de que estes efeitos podem persistir na idade adulta precoce, por exemplo, Dietrich et al. ${ }^{18}$ encontraram associações entre elevadas concentrações sanguíneas do $\mathrm{Pb}$ pré-natal e na infância e aumento das taxas de delinquência na adolescência.

Needleman et al. ${ }^{26}$ encontraram jovens com alto níveis de $\mathrm{Pb}$ no osso, tendo duas vezes mais chances de serem delinquentes, após o controle das covariáveis. Outros estudos também fazem uma ligação entre a exposição ao $\mathrm{Pb}$ e o comportamento agressivo e delinquente em adolescentes e mais tarde com violência criminal. ${ }^{18,26}$

\section{Esquizofrenla}

Um crescente corpo de evidências suporta a hipótese de que as exposições ao $\mathrm{Pb}$ podem perturbar o desenvolvimento do SNC, e estão associadas com esquizofrenia e transtornos de psicoses relacionados. ${ }^{35}$

O risco de esquizofrenia e transtornos do espectro psicótico na idade adulta mostrou ser aproximadamente o dobro em indivíduos com $\mathrm{Pb}$ no sangue materno em níveis $>15 \mathrm{mg} / \mathrm{dL}$ [indicado por níveis elevados de ácido-aminolevulínico (ALA)] durante o segundo trimestre, embora a amostra seja pequena demais para tirar conclusões definitivas. Existem razões para suspeitar que os agentes químicos em geral e, particularmente aqueles associados à industrialização, pode aumentar o risco de esquizofrenia. $\mathrm{Pb}$ é um produto tóxico amplamente reconhecido desenvolvimento, levando a prejuízos cognitivos, comportamentais e psíquicos. ${ }^{36}$

Estudos prospectivos em várias partes do mundo são consistentes em concluir que uma maior exposição ao $\mathrm{Pb}$ durante o desenvolvimento intelectual está associada com déficit cognitivo em crianças de até 10 anos de idade ${ }^{8}$.

Como múltiplas linhas de evidência de epide- 
miologia convergem, elas podem oferecer novas metas para a investigação translacional. Em alguns estudos, foram utilizadas provas sorológicas para testar a hipótese de que o desenvolvimento precoce de uma exposição ao $\mathrm{Pb}$ foi associada com aumento do risco de transtornos psicóticos em fases posteriores da vida. Os mecanismos biológicos compatíveis com os nossos resultados e com evidências clínicas e epidemiológicas disponíveis podem ainda ser investigados utilizando modelos animais de exposição e de doença, concebida especificamente para testar potencial comum no desenvolvimento da esquizofrenia e nas psicoses. ${ }^{36}$

\section{Conclusão}

Há evidências de que o cérebro imaturo é um dos mais importantes alvos, e as pesquisas apontam para consequências mais tardias, em processos cérebro degenerativos e comportamentais no adulto. Os mecanismos biológicos descritos nas exposições ao $\mathrm{Pb}$ podem perturbar o desenvolvimento do $\mathrm{SNC}$ e com evidências clínicas e epidemiológicas disponíveis precisam ser entendidos prevenindo alterações na relação estrutura-função e o desenvolvimento de psicopatologias.

\begin{abstract}
In virtually every type of exposure to lead, considering the short term or long term in high or low concentrations, the critical target organ is always the brain, encouraging signs and symptoms such as headache, memory loss, loss of concentration and attention in tasks occurring, changes in mood, with irritability, depression, insomnia (or excessive sleepiness). Several studies have shown evidence that, even at relatively low exposure levels, lead $(\mathrm{Pb})$ was detected associated with deficits in cognitive functions, intellectual and executive functioning, personality disorder like antisocial behavior and attention-deficit hyperactivity deficit disorder. Other studies were able to demonstrate that reducing the levels of exposure to $\mathrm{Pb}$ can be responsible for reducing levels of impulsivity, aggression and crime among adolescents. Finally evidence supporting the hypothesis that exposure to $\mathrm{Pb}$ can disrupt the development of the central nervous system and are associated with schizophrenia and related psychotic disorders. Some studies that reported a relationship between structure-function and psychopathology are are also discussed in this review.
\end{abstract}

Keywords: Lead/Density Factor. Cognitive Performance. Mental Disorders. Psychopathology/Behavior.

\section{Referências Bibliográficas}

1. Tong S, Lu Y. Identification of confounders in the assessment of the relationship between lead exposure and child development. Ann Epidemiol. 2000; 11:38-45.

2. Needleman HL. The neurobehavioral consequences of low lead exposure in childhood. Neurobehav Toxicol Teratol. 1982; 4:729-32.

3. Needleman HL, Gunoe C, Leviton A, et al. Deficits in psychologic and classroom performance of children with elevated dentine lead levels. N Engl J Med. 1979; 300:689-95.

4. Needleman HL, Schell A, Bellinger DC, et al. The long-term effects of exposure to low doses of lead in childhood. An 11year follow-up report. N Engl J Med. 1990; 322:83-8.

5. Needleman HL. Salem comes to the National Institutes of Health: notes from inside the crucible of scientific integrity. Pediatrics. 1992; 90:977-81.

6. Schoen EJ. Childhood lead poisoning: definitions and priorities. Pediatrics. 1993; 91:504-5.

7. Silbergeld EK. Annotation: protection of the public interest, allegations of scientific misconduct, and the Needleman case. Am J Public Health. 1995; 85:165-6.
8. Lanphear BP, Hornung R, Khoury J, et al. Low-level environmental lead exposure and children's intellectual function: an international pooled analysis. Environ Health Perspect. 2005; 113:894-9.

9. Chiodo LM, Jacobson SW, Jacobson JL. Neurodevelopmental effects of postnatal lead exposure at very low levels. Neurotoxicol Teratol. 2004; 26:359-71.

10. Canfield RL, Henderson CR Jr, Cory-Slechta DA, et al. Intellectual impairment in children with blood lead concentrations below 10 microg per deciliter. N Engl J Med 2003; 348:151726.

11. Lanphear BP, Dietrich $\mathrm{K}$, Auinger $\mathrm{P}$, et al. Cognitive deficits associated with blood lead concentrations, $10 \mathrm{microg} / \mathrm{dL}$ in US children and adolescents. Public Health Rep. 2000; 115: 521-9.

12. Mendelsohn AL, Dreyer BP, Fierman AH, et al. Low-level lead exposure and behavior in early childhood. Pediatrics. 1998; 101: E10.

13. Tong $S$, Baghurst $P$, McMichael $A$, et al. Lifetime exposure to environmental lead and children's intelligence at $11-13$ years: the Port Pirie cohort study. BMJ. 1996; 312:1569-75. 
14. Nevin R. How lead exposure relates to temporal changes in $I Q$, violent crime, and unwed pregnancy. Environ Res. 2000; 83: 1-22.

15. Nevin R. Understanding international crime trends: the legacy of preschool lead exposure. Environ Res. 2007; 104: 31536.

16. Needleman HL, Riess JA, Tobin MJ, et al. Bone lead levels and delinquent behavior. JAMA. 1996; 275: 363-9.

17. Needleman HL, McFarland C, Ness RB, et al. Bone lead levels in adjudicated delinquents. A case control study. Neurotoxicol Teratol. 2002; 24:711-7.

18. Dietrich KN, Ris MD, Succop PA, et al. Early exposure to lead and juvenile delinquency. Neurotoxicol Teratol. 2001; 23:511-8.

19. Bush G, Luu P, Posner MI Cognitive and emotional influences in anterior cingulate cortex. Trends Cogn Sci. 2000; 4: 215-22.

20. Braver TS, Barch DM, Gray JR, Molfese DL, Snyder A Anterior cingulate cortex and response conflict: effects of frequency, inhibition and errors. Cereb Cortex. 2001; 11: 825-36.

21. Williams ZM, Bush G, Rauch SL, Cosgrove GR, Eskandar EN Human anterior cingulate neurons and the integration of monetary reward with motor responses. Nat Neurosci. 2004; 7: 1370-5.

22. Davis KD, Taylor KS, Hutchison WD, Dostrovsky JO, McAndrews MP, et al. Human anterior cingulate cortex neurons encode cognitive and emotional demands. J Neurosci. 2005; 25: 8402-6.

23. Ris MD, Dietrich KN, Succop PA, Berger OG, Bornschein RL Early exposure to lead and neuropsychological outcome in adolescence. J Int Neuropsychol Soc. 2004;10: 261-70.

24. Froehlich TE, Lanphear BP, Dietrich KN, Cory-Slechta DA, Wang N, et al. Interactive Effects of a DRD4 Polymorphism, Lead, and Sex on Executive Functions in Children. Biol Psychiatry. 2007, 62: 243-9.

25. Sciarillo WG, Alexander G, Farrell KP. Lead exposure and child behavior. Am J Public Health. 1992; 82: 1356-60.

26. Needleman HL, Riess JA, Tobin MJ, Biesecker GE, Greenhouse JB. Bone lead levels and delinquent behavior. JAMA. 1996; 275: 363-9.
27. Brockel B.J., Cory-Slechta D.A., Lead, attention, and impulsive behavior: Changes in a fixed-ratio waiting-for-reward paradigm. Pharmacol Biochem Behav. 1998; 60 545-52.

28. Faust, J. Brown, Moderately elevated blood lead levels: Effects on neuropsychologic functioning in children, Pediatrics.1987; 80: 623-9.

29. Silva PA, Hughes P, Williams S, Faed JM. Blood lead, intelligence, reading attainment, and behaviour in eleven year old children in Dunedin, New Zealand. J. Child Psychol Psychiatry.1988; 29: 43-52.

30. Froehlich TE, Lanphear BP, Dietrich KN, Cory-Slechta DA, Wang N, et al. Interactive Effects of a DRD4 Polymorphism, Lead, and Sex on Executive Functions in Children. Biol Psychiatry. 2007; 62: 243-9.

31. Braun JM, Kahn RS, Froehlich T, Auinger P, Lanphear BP Exposures to environmental toxicants and attention deficit hyperactivity disorder in US. children. Environ Health Perspect. 2006; 114: 1904-9.

32. Lanphear BP, Dietrich KN, Auinger P, Cox C, Cognitive deficits associated with blood lead concentrations $<10$ microg/dL in US children and adolescents. Public Health Rep. 2000; 115: 521-9.

33. Bellinger D, Leviton A, Allred E, Rabinowitz M. Pre- and postnatal lead exposure and behavior problems in school-aged children. Environ. Res. 1994; 66: 12-30.

34. Mendelsohn AL. et al Low-level lead exposure and behavior in early childhood. Pediatrics.1998;101-10.

35. McGrath J, Eyles D, Mowry B, Yolken R, Buka S. 2003. Low maternal vitamin $D$ as a risk factor for schizophrenia: a pilot study using banked sera. Schizophr Res. 2003; 63:73-8.

36. Opler MG, Buka SL, Groeger J, McKeague I, Wei C, FactorLitvak P, Bresnahan M, et al. Prenatal exposure to lead, deltaaminolevulinic acid, and schizophrenia: further evidence. Environ Health Perspect. 2008;116: 1586-90. 